\title{
A New Strange Nonchaotic Attractor-like
}

\author{
Lin Mei \\ Air Force Engineering University Aeronautics and \\ Astronautics Engineering College \\ Xi'an, Shaanxi, China \\ E-mail: 510486495@qq.com
}

\author{
Song Guangshun \\ Xi'an Municipal Engineering \& Research Institute Co. , \\ Ltd. \\ Xi'an, Shaanxi, China \\ E-mail:64695587@qq.com
}

Liu Yongqiang

Air Force Engineering University Aeronautics and Astronautics Engineering College

Xi'an, Shaanxi, China

\author{
Zhu Yanbo \\ Air Force Engineering University Aeronautics and \\ Astronautics Engineering College \\ Xi'an, Shaanxi, China \\ Zhang Xiaolu \\ Air Force Engineering University Aeronautics and \\ Astronautics Engineering College \\ Xi'an, Shaanxi, China
}

\begin{abstract}
Strange nonchaotic attractors exist in quasiperiodically forced system universally, which have fractal structure in geometry but no sensitivity to initial value, which also are among the international hotspot issues studied by nonlinear kinetics for the recent ten years. Many scholars have done a lot of research and made gratifying achievements, evolved process and mechanism of which should be further studied and explored on the hand. Two kinds of quasiperiodically forced circuit system and time-lagged Logistic system are studied in this paper. From numerical simulation by changing parameters in the system, a new type of SNA shaped "tire " or "conduit ", evolved from $T^{2}$ torus bifurcation is found, being distinguished from others for its wide range of parameter space and fractal phase space structure of a particular shape. The study indicates that this attractor exists in general Neimark-Sacker bifurcation system. The study also brings new phenomena of dynamical system to light, suggesting its wide application prospects.
\end{abstract}

Keywords-Strange nonchaoitc attractors; T2 torus bifurcation; Neimark-Sacker bifurcation; Lyapunov exponents; Time delayed logistic equation

\section{PREFACE}

Strange nonchaotic attractors exist in quasiperiodically forced system universally, Which have fractal structure in geometry but has no sensitivity to initial value. SNAs are among the international hotspot issues studied by nonlinear kinetics for the recent ten years. Many domestic and foreign scholars have done a lot of research and made gratifying achievements mainly from the numerical simulation of SNAs ever since such scholars as Grebogi and Ott proposed the conception of SNAs $^{[1]}$, whose research concentration was on dynamical transfer ${ }^{[2-4]}$, evolved mechanism ${ }^{[5-8]}$ and attractor crisis ${ }^{[9-12]}$ about SNAs. SNAs has shown a lot of similar characteristics to chaotic attractor, which has fractal structure of the phase space as an example. On the other hand, SNAs are different from the chaotic attractors, which have wide application prospect in security communication fields ${ }^{[13-15]}$. Research to evolved process and mechanism of SNAs become the focus attention of many scholars, which mainly includes: The saddle node bifurcation intermittent-I, Subharmonic bifurcation intermittent-III, Torus collision, Torus fractal and attractor crisis etc. However, evolved process and mechanism of which should be further studied and explored.

Two kinds of quasiperiodically forced Neimark-Sacker bifurcation system are studied in this paper. Except for the five typical SNAs found in the past ten years, quasiperiodically forced Neimark-Sacker bifurcation system, another SNAs shaped as "tire-shaped" or "pipe-shaped", evolved from $\mathrm{T}^{2}$ torus bifurcation, shape of which depends on the shape of torus quasiperiodically before bifurcation. This type of SNAs distinguished from other SNAs for its wide range of parameter space and fractal phase space structure of a particular shape. From the study of geometrical characteristics of SNAs, new phenomenon in dynamic systems is further revealed, application prospects of which is broad.

\section{BIFURCATION STRUCTURE AND SNAS OF CIRCUITS}

A kind of circuit model is provided like the following, state equation of which is as equation 1 ,

$$
\left\{\begin{array}{l}
\mu_{n+1}=\frac{R}{R_{\omega}} v_{n}-\frac{0.1 R}{R_{3}} v_{n}{ }^{2} \\
v_{n+1}=-\left(1+\frac{R_{1}}{R}\right) \mu_{n}+\frac{R}{R_{2}} v_{n}
\end{array}\right.
$$


Dimensionless transformation is written as $x_{n}=\mu_{n}$, $y_{n}=v_{n}, a=R / R_{\omega}, d=0.1 R / R_{3}, b=1+R_{1} / R, c=R / R_{2}$, equation 1 can be changed to equation 2 ,

$$
\left\{\begin{array}{l}
x_{n+1}=a y_{n}-d y_{n}{ }^{2} \\
y_{n+1}=-b x_{n}+c y_{n}
\end{array}\right.
$$

By adding the driver of quasiperiodically forced system, equation 2 can be written as,

$$
\left\{\begin{array}{l}
x_{n+1}=p \cos \left(2 \pi z_{n}\right)+a y_{n}-d y_{n}^{2} \\
y_{n+1}=-b x_{n}+c y_{n} \\
z_{n+1}=z_{n}+\omega(\bmod 1)
\end{array}\right.
$$

$\omega$ is a irrational number, $p$ is the stress amplitude of vibration.

System 3 can be regarded as a disturbance system of system 2, either of which has a variety of bifurcation structure.

Make $A=c^{2}-4 a b, \quad B=c^{2}-8 c+4 a b+8, \quad$ pitchfork bifurcation appears at $p 1$ when $A \geq 0$ and c $+\sqrt{A}=2$. $a b=1$.

Neimark-Sacker bifurcation appears when $A<0$ and

Particularly, subharmonic bifurcation under the condition of four times of strong resonance appears when $c=0$ and $a b=1$.

Pitchfork bifurcation appears at $p 2$ when $B \geq 0$, $c+\sqrt{B}=2$ and $c<2$.

Neimark-Sacker bifurcation appears when $B<0$ and $2 c-a b=3$.

For the reason of quasiperiod, period $\mathrm{n}$ in system 3 changes to $\mathrm{nT}$ torus when the driver of quasiperiodically is added.

Bifurcation position may delay or predict when amplitude of driving force changes.

Relevant dimensionless parameter can be obtained from different values of $R, R_{\omega}, R_{1}, R_{2}$ and $R_{3}$

From their physical meanings, we know that all the parameters $a, c, d$ and $p$ are greater than zero.

For system 3, when $b>1, \omega$ can be valued as the golden ratio constant $(\sqrt{5}-1) / 2, b=1.1, c=0.1, d=0.2, p$ and $a$ are both variable parameters.

Fig .1(a) and $1(b)$ give us the bifurcation diagrams expressing the changes of $X n$ when the variable parameters $a \in(0.6,1.6)$ changes, and the maximum Lyapunov exponents figure $(\lambda \max )$.

Upon analysis, conclusion can be made that the system is of quasiperiodical motion, phase plane of which appears 1T torus.

For system 2, when $a \approx 0.9091$, Hopf bifurcation $a=1 / b \approx 0.9091$, bifurcation $\mathrm{T}^{2}$ torus appears and the system enters region SNAs, the maximum Lyapunov exponents figure $(\lambda \max )$ is less than zero, for some individual sites are almost equal to zero.

Phase plane in Fig. $1(c)$ presents such shape as oval bottle, tire, etc.

One type of fixed point is given in Fig .2(a) three types of SNAs are presented in Fig .2(b), Fig .2(c) and
Fig .2(d).

Until $a \approx 1.1728,4 \mathrm{~T}$ torus appears because of quasiperiodical subharmonic bifurcation.

Persistent $4 \mathrm{~T}$ torus is presented in Fig .2(d) when $a=1.173$. The system changes to chaotic motion and Hyper-chaotic motion, being evolved from other types of SNAs when $a$ rises.

Similar SNAs appears when the parameter $a$ is fixed at $a=0.93$ and $p$ at $p \in(0.01,0.668)$, the maximum Lyapunov exponents figure $(\lambda \max )$ is presented in figure $1(d)$.

The maximum Lyapunov exponents figure $(\lambda \max )$ is no greater than zero within this interval, presenting nonchaotic strange attractors. Similar SNAs structure is presented in Fig .2(e), which is tube-shaped when $p=0.65$.

The system comes to quasiperiodical motion and phase structure of which appears $1 \mathrm{~T}$ torus when $p>0.668$. Torus attractors of $1 \mathrm{~T}$ torus when $p=0.675$ is presented in Fig .2(f). Attractors shown in Fig .2(e) is from this torus bifurcation, appear SNAs ringing as the axis of this ring.

From the above analysis, we know that SNAs appears in larger parameter selection intervals, making either $a$ or $p$ as variable parameter.

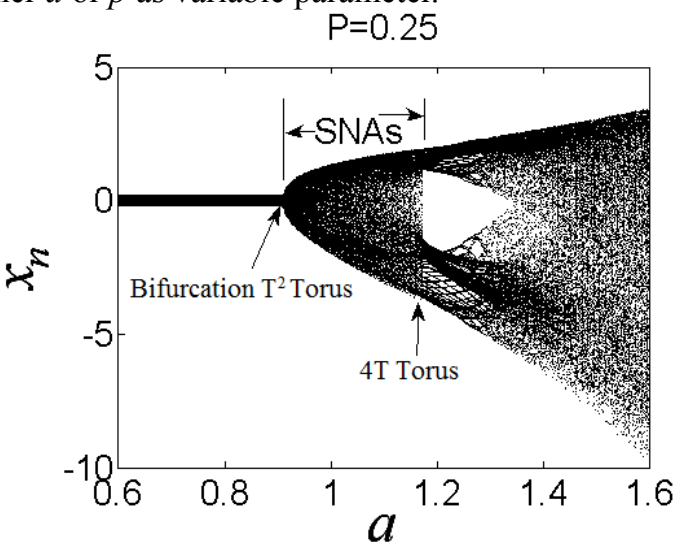

Figure 1(a)

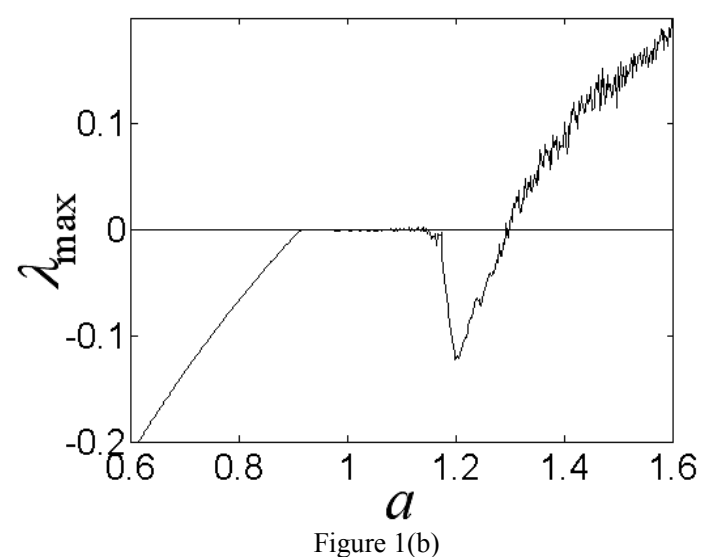

Figure 1(b) 

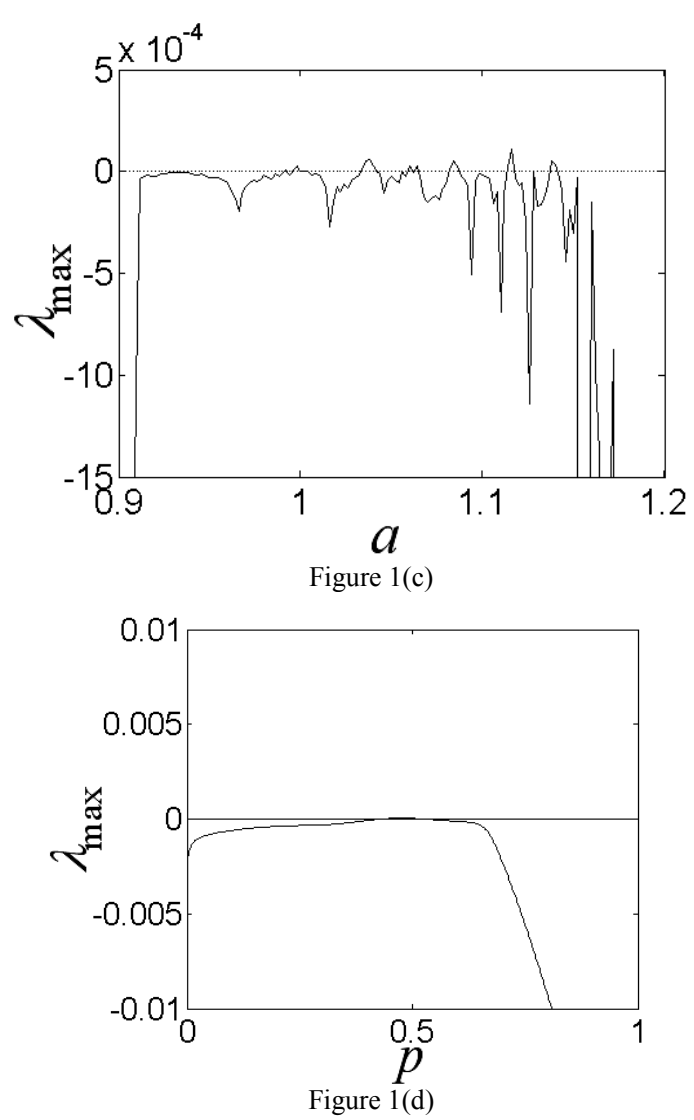

Figure 1 The Bifurcation Diagrams and the Maximum Lyapunov Exponents Figure $(\lambda \max )$

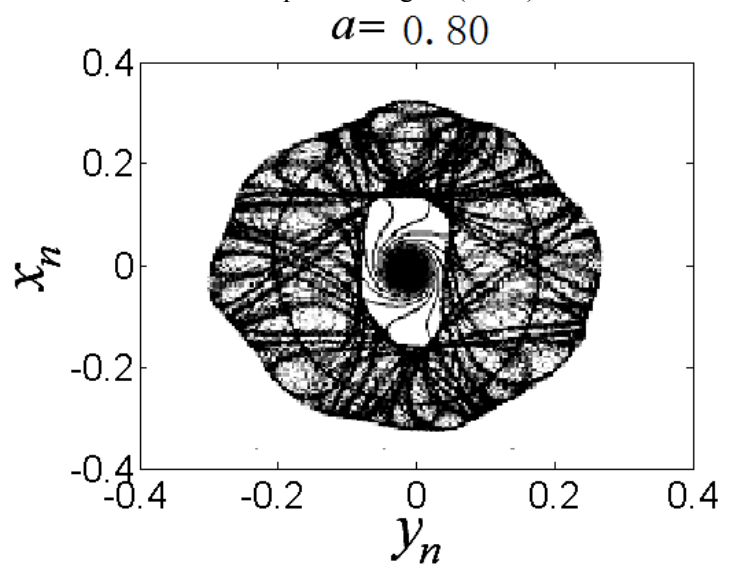

Figure 2(a) Fixed Point a

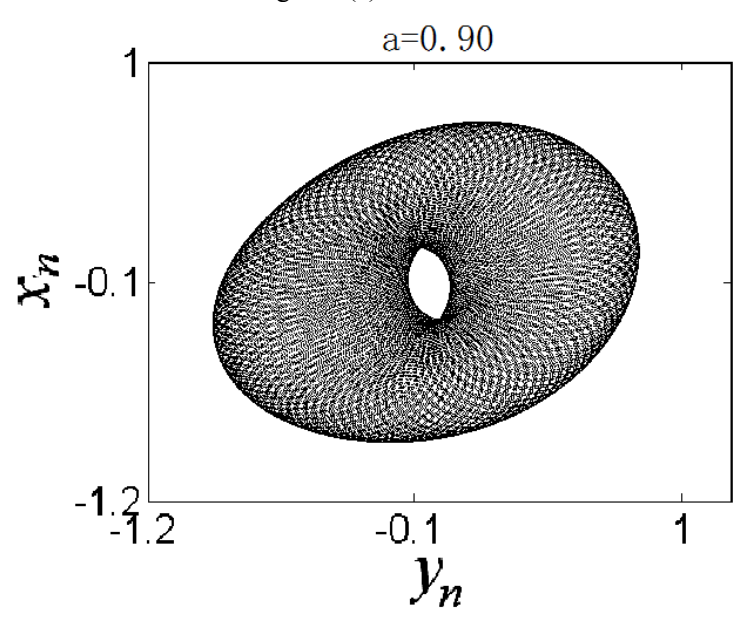

Figure 2(b) SNAs Type a

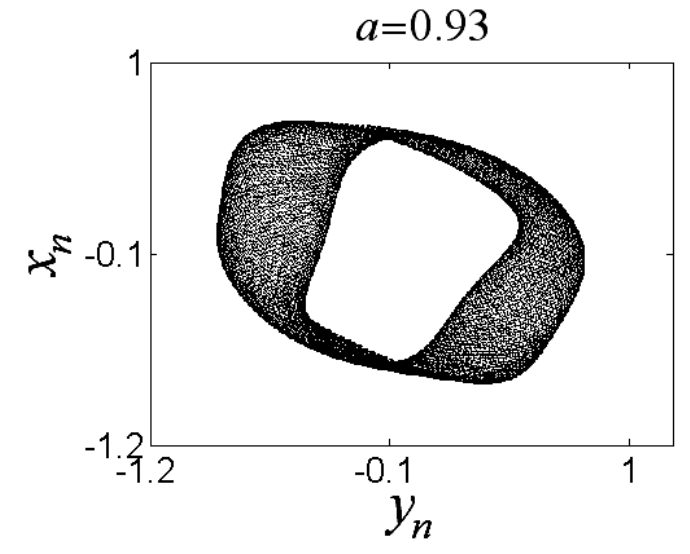

Figure 2(c) SNAs Type b

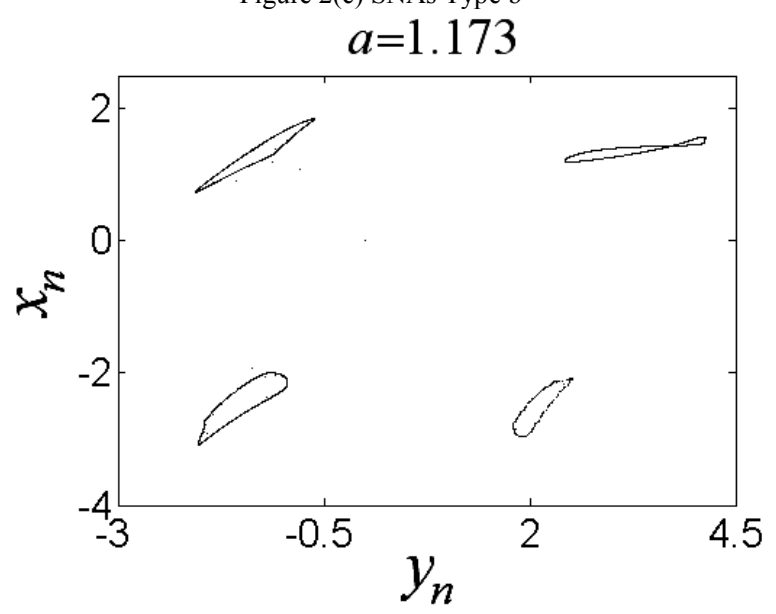

Figure 2(d) $4 \mathrm{~T}$ Torus

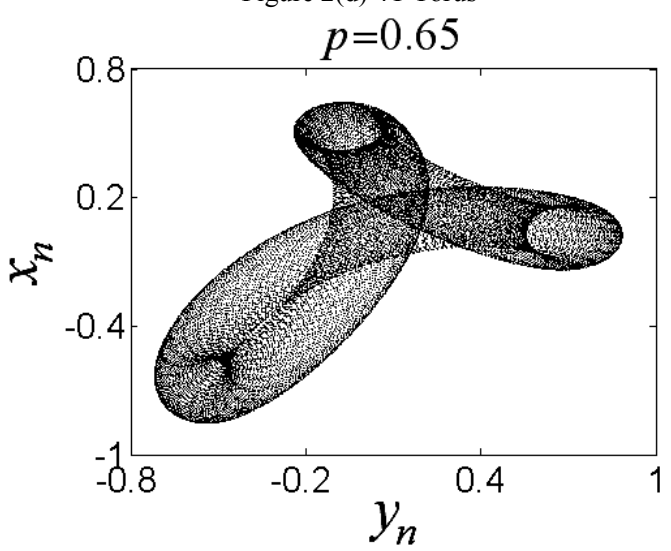

Figure 2(e) SNAs Type e

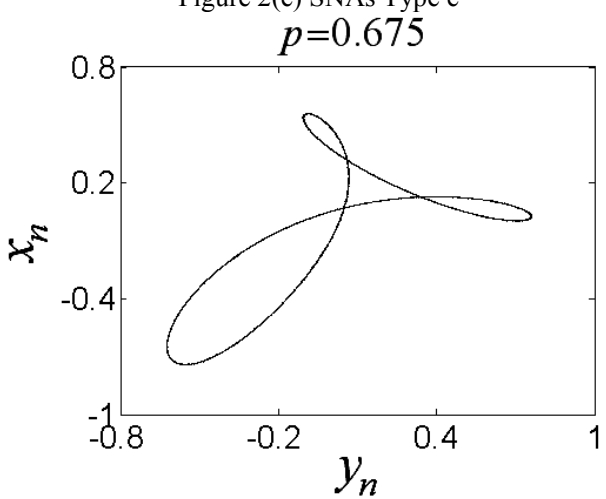

Figure 2(f) 1T Torus

Figure 2, Torus Bifurcation Mechanism 


\section{TIME DELAYED LOGISTIC EQUATION AND SNAS}

Classic state equations of Logistic model is as the following,

$$
x_{n+1}=\mu x_{n}\left(1-x_{n-1}\right)
$$

In the upper equation, $\mu$ is for growth rate, $x_{n}$ is for density of species-group or population due to time. Through theoretical deduction and calculation, we know that the system lose stability and Neimark-Sacker bifurcation appears when $\mu=\mu_{0}=2$ at the fixed point of $\left(1-1 / \mu, 1-1 / \mu_{0}\right)$.

$\left\{\begin{array}{l}x_{n+1}=\mu\left(\varepsilon+p \cos \left(2 \pi z_{n}\right)\right) x_{n}\left(1-x_{n-1}\right) \\ z_{n+1}=z_{n}+\omega(\bmod 1)\end{array}\right.$

Value $\omega$ as golden ratio as constant $(\sqrt{5}-1) / 2$, $\varepsilon=1.8, p=0.2$ and $\mu$ as varying parameter.

Bifurcation of $X n$ that varies from $\mu \in(0.4,1.25)$ is present in Fig.3.

Confirmatory analysis shows that the system appears periodic motion and phase plane appears $1 \mathrm{~T}$ torus when $\mu \approx 0.556$.

Confirmatory analysis also shows that the system appears bifurcation torus $T^{2}$ and the system enters the SNAs area, maximum Lyapunov exponents being less than zero and some being near to zero, phase plane of which presents some special geometric configuration.

Fig .3(b) presents tire-shaped SNAs when $\mu=1.13$. Fig $.3(c)$ and $3(d)$ present the two typical SNAs separately when $\mu=1.13, p=0.2$ and $\mu=2.1, p=0.385, \varepsilon=1$.

Torus fractal is not torus collision but a new type of SNAs appears when the quasiperiodical torus loses its smoothness for sudden extreme distortion of which following the change of parameters. Usually, torus fractal studied includes single torus fractal and $2^{n}$ torus fractal. $\mathrm{T}$ torus fractal appears in this circuitry studied, single torus fractal appears easily when is $p$ larger. A new type of SNAs appears when torus doubling ring get extremely distorted because of interruption of torus doubling ring.

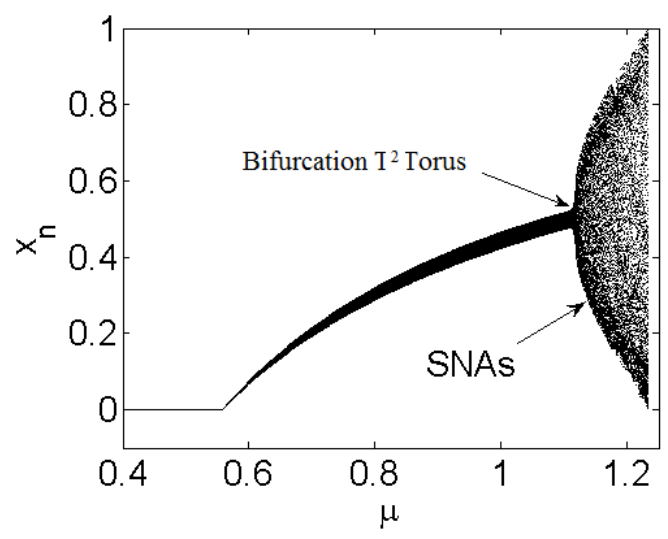

Figure 3(a) SNAs Type a

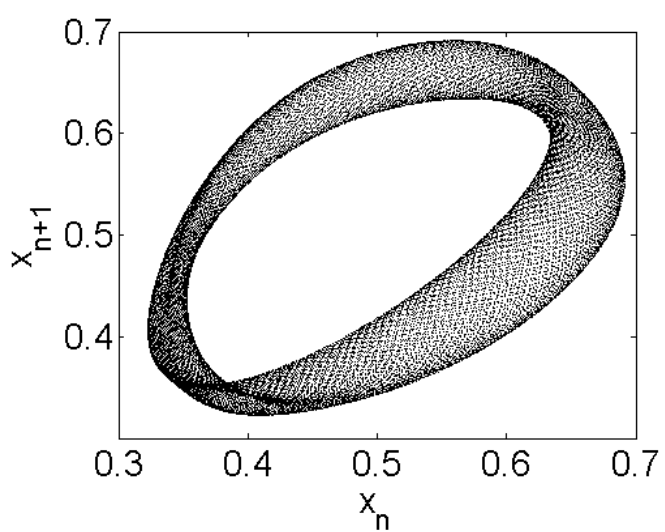

Figure 3(b) SNAs Type b

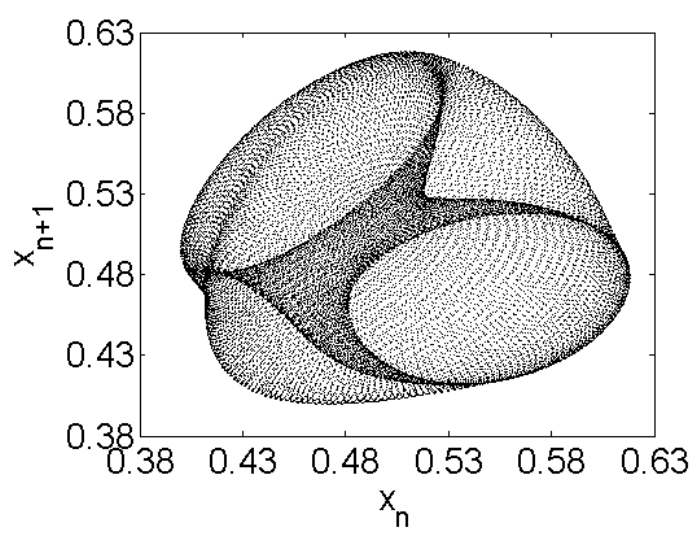

Figure 3(c) SNAs Type c

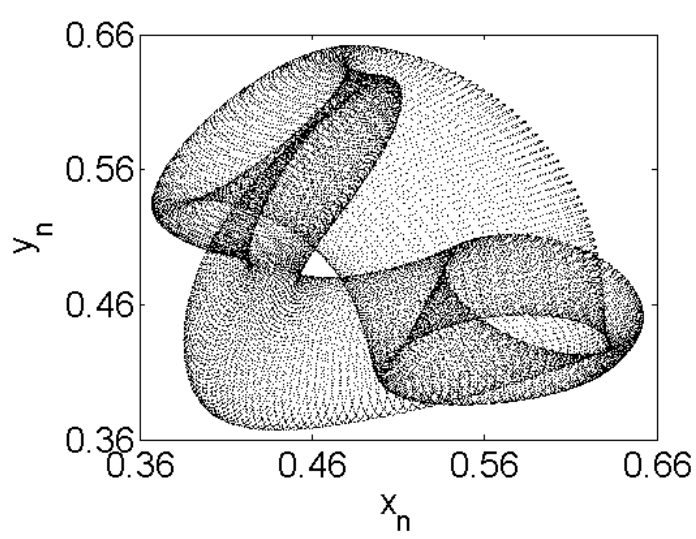

Figure 3(d) SNAs Type d

Figure 3 Bifurcation Torus T2 and SNAs

\section{CONCLUSIONS}

From studying the two types of quasiperiodically forced Neimark-Sacker Bifurcation system, a new type of SNAs evolved from $\mathrm{T}^{2}$ torus bifurcation is found in this paper, geometric structure of which appears typical geometric shape. Numerical simulation and analysis are also used to reveal nonlinear dynamical phenomenon and more complicated dynamical routine from various aspects in this paper. This type of attractor exists in large scale parameter intervals.

The study indicates that production of SNAs has affinity with bifurcation, which appears in torus doubling bifurcation, $\mathrm{T}^{2}$ torus bifurcation, subharmonic bifurcation and saddle-node bifurcation, etc. SNAs is described by 
maximum Lyapunov exponents. The study indicates that this routine and mechanism is very universal and SNAs has great application prospects.

For SNAs, such aspects as geometric dimension, type and mechanism of SNAs in periodically forced system should be further studied to cognize dynamic behavior and operating mechanism of vibration system. This research can provide theoretical reference and new idea for bug diagnosis, vibration control, safe operation and malfunction prediction in actual industry control system.

\section{REFERENCES}

[1] C. Grebogi, E. Ott, S. Pelikan, and J. A. Yorke, Physica D 13,261(1984).

[2] T. Yalcinkaya, Y.C. Lai, Phys. Rev. Lett. 77, 5039 (1996).
[3] A. Prasad, V. Mehra, R. Ramaswamy, Phys. Rev. Lett. 79, 4127 (1997).

[4] T. Nishikawa, K. Kaneko, Phys. Rev. E 54, 6114 (1996).

[5] J. F. Heagy, S. M. Hammel, Physica D 70, 140 (1994).

[6] B.R.Hunt, E.Ott, Phys. Rev. Lett. 87, 254101 (2001).

[7] J. W. Kim, S.-Y. Kim, B. Hunt, E. Ott, Phys. Rev. E 67, 036211 (2003).

[8] P. Alstrom, B.Christiansen, M.T. Levinsen, Phys. Rev. Lett. 61, 1679 (1988).

[9] A. Prasad, R. Ramaswamy, Phys. Rev. Lett. 83, 4530 (1999).

[10] H. M. Osinga, U. Feudel, Physica D 141, 54 (2000).

[11] J. J. Stagliano, J.-M. Wersmger, E. E. Slaminka, Physica D 92, 164 (1996).

[12] S. S. Negi, A. Prasad, R. Ramaswamy, Physica D 145, 1 (2000).

[13] C. S. Zhou and T. L. Chen, Europhys. Lett. 38, 261(1997).

[14] R. Ramaswamy, Phys. Rev. E. 56, 7294 (1997).

[15] R. Chacon and A. M. Gracia-Hoz, Europhys. Lett. 57, 7 (2002). 